\title{
Antimicrobial Resistance Profile among clinical isolated bacteria at Wolaita Sodo University Teaching Referral Hospital, three year retrospective cohort study
}

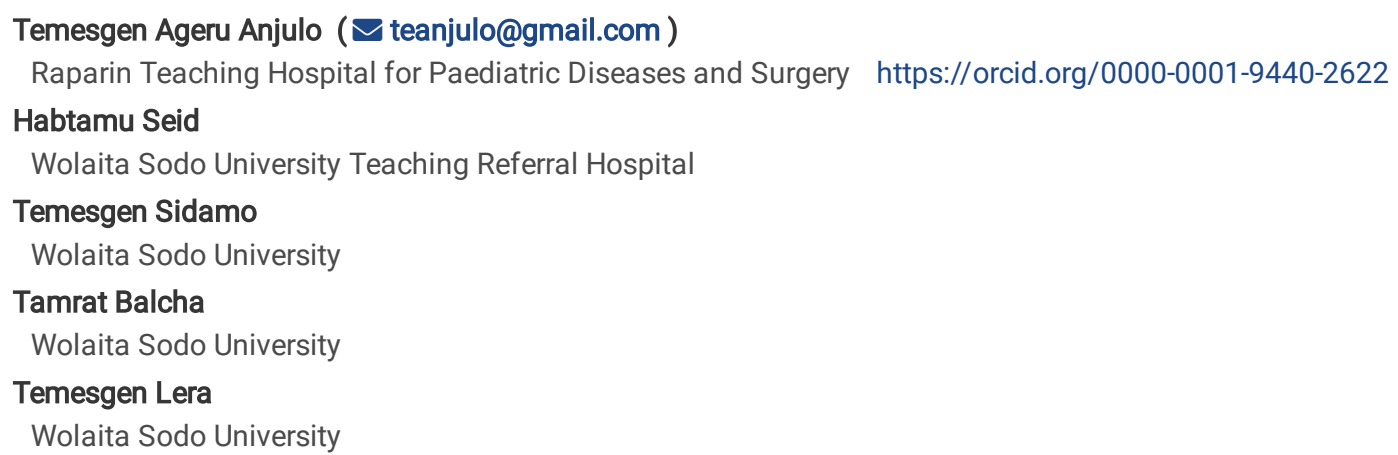

Research

Keywords: Bacteria, Antibiotic resistance, Wolaita Sodo University

Posted Date: April 24th, 2020

DOI: https://doi.org/10.21203/rs.3.rs-23586/v1

License: @ (i) This work is licensed under a Creative Commons Attribution 4.0 International License. Read Full License 


\section{Abstract \\ Background}

Antibiotic resistance is public threats in globally and its problem not well concerned in sub Saharan Africa including Ethiopia due to inadequate set up. However, there is information gap in resistance of antibiotic and its pattern for physicians who prescribe. Therefore, the aim of this study was to review the antimicrobial resistance pattern of bacterial isolated from different specimens inoculated retrospectively at Wolaita Sodo University Teaching Referral Hospital, Southern Ethiopia.

\section{Methods}

A 3 year retrospective data of culture and antibiotic sensitivity pattern analyzed from 2016-2018 among 330 patients records at Wolaita Sodo university Teaching Referral hospital. The data extracted were clinical samples taken, age, sex, bacteria growth status, bacteria isolated, antibiotic resistance profile and trend in each year.The data entry was done using Epidata version 3.5 .1 and exported to SPSS version 21 software for cleaning and statistical analysis.Bivariable analysis was done and variables with p-value of less than 0.25 were made candidate for multiple logistic regression analysis. The relative contribution of each selected variables to the outcome of interest was assessed using multiple logistic regression and variables with P-value less than 0.05 were considered as statically significant.

\section{Results}

A total of 330 samples fulfills inclusion criteria were extracted from microbiology laboratory, 150 samples were become culture positive for bacteria isolates. From positive culture growth, 110 (73.3\%) was gram positive bacteria and $40(26.7 \%)$ gram negative. A staphylococcus auras (S.auras) was the most prevalent isolate 97 (61.3\%) from gram positive isolates and Escherichia coli (E. coli) 19(12.7\%) was gram negative. From gram positive isolates S. auras was $53 \%$ and S. saprophytes $62 \%$, S. pyogen $74 \%$ and S.pneumonia $52 \%$ resistance to antibiotics. Overall resistance for all antimicrobial resistance of gram negative of E. coli was $62 \%$, P. aurogenous $75 \%$, proteusspp $65 \%$, shigellaspp $66 \%$, salmonella $56 \%$ and NisseriaSpp 63\%.Overall antimicrobial resistance of gram positive isolates was $54.2 \%$ gram negative bacteria was $60.0 \%$

\section{Introduction}

Antibiotic resistance is a major threat to health and human development affecting our ability to treat a range of infectious microorganisms [1]. AMR is the ability of Microorganism to resist antimicrobials which may happen naturally, but misuse of antibiotics in humans and animals, for inappropriate microorganisms. As consequence, the antibiotics become ineffective and infection continues in the body, increasing the risk of multiply to person to person of [2]. Resistance to antibiotics occurs through modified antimicrobial target, enzymatic hydrolysis, the outflow of cell membrane and impermeability. Multi antibiotic resistance bacteria acquire resistance by mutation, and gene transfer via conjunctions, transformation, or transduction by which treatment becomes difficult[3]. The expenditure of health care for patients with resistant infections is higher than care for patients with non-resistant infections because of longer duration of illness, additional tests and the need for more expensive medicines. [4] The rise in resistance not only impedes our ability to treat infections, but has broader societal and economic effects, and endangers the achievement of the Sustainable Development Goals [5, 6]. The direct and indirect impact of AMR will mostly fall on low- and middle-income countries, which often lack the infrastructure, and human and financial resources to adequately counter drug resistance epidemics [6]. The consequences of AMR are aggravated in volatile situations such as civil unrest, violence, famine and natural disasters, as well as in settings with poor health care services or without access to health care [5, 7]

AMR is a major public health concern in both developed and developing countries and it can affect anyone, any age, both sex, any country. AMR is high prevalent in low and middle in because of high prevalent of infectious disease, lack of trained health professionals, irrational use of drugs and limited set up of microbiological laboratory[4, 8].

According to WHO report of 2014, five out of six WHO regions had more than $50 \%$ resistance to third generations Cephalosporin and fluoroquinolones in Escherichia coli and methicillin resistance in Staphylococcus auras in hospital setting. The report attributed 45\% of deaths in both Africa and South-East Asia to multi-antibiotic resistant bacteria. It further revealed that K. pneumonia resistant to third generation cephalosporin was associated with elevated deaths in Africa (77\%), the Eastern Mediterranean region (50\%), South East Asia (81\%)and Western Pacific region(72\%)[[8]. According to death attributed in 2016 of AMR in Asia was 4,730,000 and that of Africa was 4,150,000[9].

Surveillance provides data that can be easily compared, exchanged or used locally, nationally and globally. Unfortunately, many low- and middle income countries including Ethiopia lack the capacity to establish and maintain systems to collect and make use of data on antimicrobial consumption.

Thus, obtaining periodical estimates of the local epidemiological picture of antimicrobial resistance by monitoring various risk groups is essential for guiding clinical action, resource allocation, and intervention protocols to make clinical staff aware. Since start of culturing and antimicrobial 


\section{Methods \\ Study setting and design}

The study was conducted at Wolaita Sodo University Teaching Referral Hospital (WSUTRH), which is located in Wolaita Zone, 154 km from Hawassa, capital of South nations, nationalities and peoples region, and $329 \mathrm{~km}$ from Addis Ababa, capital of Ethiopia. Currently it provides both patient care and teaching services. The hospital established 1928 and it is the oldest and largest hospital. In 2014 it was incorporated to Wolaita Sodo University, since then it services as both teaching and patient care. The hospital provides general outpatient and inpatient services including medical, surgical, pediatric, psychiatric, Ophthalmic, gynecology and Obstetrics Emergency care. Annual patient volume is around 200,000 and has 370 beds. It provides well organized laboratory services in both academic and Clinical wings. Clinical laboratory has also five units, central laboratory unit, Emergency and critical care laboratory, inpatient laboratory, maternal and child health laboratory, outpatient services laboratory. Microbiology laboratory is one part of Central laboratory in which Culture and antimicrobial testing conducted and it was charged by chief Medical microbiologists.

\section{Study design and period:}

Institutional based three year retrospective study from 2016-2018 data collected from June 20/2019-July 20/2019

\section{Source Population}

Data of all patients attended Wolaita Sodo University Teaching Referral Hospital, South Ethiopia during 2016-2018 were included.

\section{Study Population:}

Patients who requested culture and gave sample for microbiological data during 2016-2018 fulfill inclusion criteria.

\section{Inclusion criteria and Exclusion criteria}

All completed patient data with full information registered in the logbook during the study period was included and patient data with incomplete data were excluded

\section{Data collection:}

Demographic data of patients, the bacteria isolated and the antimicrobial susceptibility profiles were retrieved from Wolaita Sodo teaching referral University hospital (WSUTRH) microbiology, laboratory unit registration records using a standard data collection form. Laboratory records which had incomplete information of either age, sex or culture and drug susceptibility test results were excluded.

\section{Quality assurance:}

A standard bacteriological procedure was followed to maintain correct laboratory test results before testing patient sample inoculated. American Type Culture collection (ATCC) standard reference strains Escherichia coli (E. coli) ATCC-25922, Staphylococcus auras (S. auras) ATCC 25923 and Pseudomonas aeruginosa (P. aeruginosa) ATCC-25853 were used to control quality of culture and drug susceptibility testing. All data was checked for consistency and completed registration or recording document.

\section{Ethical considerations}

The ethical clearance was obtained from the Ethical review committee of Wolaita Sodo University, College of Health Science and medicine. Formal permission was also obtained from Wolaita Sodo University Referral Hospital. For reasons of privacy, all data were kept confidential. Anonymity of records was maintained by using registration number and unique code numbers used by service providers at Wolaita Sodo University teaching referral hospital.

\section{Data Processing and Analysis}

The preliminary data were checked and processed depending on the variables.Data analysis was done by using SPSS Version-20 software and results were summarized using means and percentages and presented by using graphs and tables

\section{Result}

\section{Socio demographic characteristics of the subjects}

In total 330 patients data recorded over 2016 to 2018 and those that fulfill the inclusion criteria were extracted. Based on their sex, 167 (50.6\%) were male and 163 were female (49.4\%). The majority of the patients (26.45) included in this study were in the age range of 25 to 34 years. From total 330 patients, 124(37.6\%) visited microbiological laboratory in the year of 2017 (See Table 1). 
Table 1

Socio-demographic characteristics of patients $(N=330)$ involved in Antimicrobial resistance profiling 2016 to 2018

\begin{tabular}{|llll|}
\hline Variable $(\mathbf{N}=\mathbf{3 3 0})$ & & Number & Percentage (\%) \\
\hline Sex & Male & 167 & 50.6 \\
\hline Fge group & Female & 163 & 49.4 \\
\hline & $<5$ years & 55 & 16.7 \\
\cline { 2 - 4 } & $5-14$ & 38 & 11.5 \\
\hline & $15-24$ & 66 & 20.0 \\
\hline & $25-34$ & 87 & 26.4 \\
\hline & $35-44$ & 46 & 13.9 \\
\hline Years patient visited microbiology lab & 2016 & 38 & 11.5 \\
\cline { 2 - 4 } & 2017 & 117 & 35.5 \\
\hline & 2018 & 124 & 37.6 \\
\cline { 2 - 4 } & & 89 & 27.0 \\
\hline
\end{tabular}

Table 2

Age distribution of patients from which the bacterial isolates obtained at WSUTRHL, 2016-2018.

\begin{tabular}{|c|c|c|c|c|c|c|c|}
\hline \multirow{2}{*}{$\begin{array}{l}\text { Growth status } \\
(\mathrm{N}=330)\end{array}$} & \multicolumn{6}{|c|}{ Patient age group } & \multirow[t]{2}{*}{ Total } \\
\hline & $<5$ years & $5-14$ years & $15-24$ years & $25-34$ years & $35-44$ years & 45 years and above & \\
\hline Positive culture & $26(17.3 \%)$ & $19(12.7 \%)$ & $37(24.7 \%)$ & $30(20.0 \%)$ & $17(11.3 \%)$ & $21(14.0 \%)$ & $150(100 \%)$ \\
\hline Negative & $29(16.1 \%)$ & $19(10.6 \%)$ & $29(16.1 \%)$ & $57(31.7 \%)$ & $29(16.1 \%)$ & $17(9.4 \%)$ & $180(100)$ \\
\hline Total & $55(16.7 \%)$ & $38(11.5 \%)$ & $66(20.0 \%)$ & $87(26.4 \%)$ & $46(13.9 \%)$ & $38(11.5 \%)$ & $330(100 \%)$ \\
\hline
\end{tabular}

\section{Bacterial growth status and sample inoculated at WSUTRH, 2016-2018}

Among the total 330 patient specimens inoculated, 150 (45.5\%) were found to show positive bacterial growth whereas 180 (54.5\%) didn't show any growth. In terms of the specimen types used for the bacterial isolation 159 (48.2\%) were discharges from vagina, urethra, wound 49 (14.8\%) from stool, 71 (21.5\%) from urine and 51 (15.5\%) were from other body fluids(See Table 3).

Table 3

Distribution of time BSE practiced and the reasons given to perform or not among women in Sodo city, 2019 $(n=626)$.

\begin{tabular}{|llll|}
\hline Variable $(\mathbf{N}=\mathbf{3 3 0})$ & & Number & Percentage (\%) \\
\hline Growth status & Positive & 150 & 45.5 \\
\cline { 2 - 4 } & Negative & 180 & 54.5 \\
\hline Types of Specimens used for bacterial isolation & Urine & 71 & 21.5 \\
\cline { 2 - 4 } & *Discharges and swab & 159 & 48.2 \\
\cline { 2 - 4 } & Body fluid & 51 & 15.5 \\
\cline { 2 - 4 } & Stool & 49 & 14.8 \\
\cline { 2 - 4 }
\end{tabular}

\section{Types of Bacteria's Isolated from positive growth}

The top three bacteria isolated from those positive growths in this study were S. aureus 92 (61.3\%), E.coli19(61.3\%) and S. saprophyte $9(6 \%)$. (See Table 4). 
Table 4

Prevalent of pathogenic bacteria among positive isolation at WSUTRH, 2016-2018

\begin{tabular}{|lll|}
\hline Variable $(\mathbf{n}=\mathbf{1 5 0})$ & Frequency & Percentage (\%) \\
\hline S. aureus* & 92 & 61.3 \\
\hline E.coli & 19 & 12.7 \\
\hline S. saprophytes* & 9 & 6.0 \\
\hline P. aurogenous & 8 & 5.3 \\
\hline S. pneumonia* & 5 & 3.3 \\
\hline S. pyogenes* & 4 & 2.7 \\
\hline Proteus spp. & 4 & 2.7 \\
\hline Shigella & 4 & 2.7 \\
\hline Neisseria spp. & 3 & 2.0 \\
\hline Salmonella & 2 & 1.3 \\
\hline Total & 150 & $100 \%$ \\
\hline
\end{tabular}

Among those 150 positive growths, based on their gram reaction, 110(73.3\%) were gram positive and 40(26.7\%) were gram negative.Gram positive bacteria identified were $S$. auras, $S$. pyogen, $S$, pneumonia, and $S$. saprophytes whereasgram negative bacteria isolated were, E.coli, $P$. aurogenus, ProtousSpp, salmonella spp, shigellaspp and NisseraiSpp(See Fig. 1).

\section{Antimicrobial Resistance profile among gram positive bacteria isolated}

Gram positive bacteria identified were $S$. auras, S. saprophytes, $S$. pyogen and S. pneumonia. The overall antimicrobial resistances for $S$. aureus, $S$. saprophytes, S. pyogen, and S. pneumonia were rated as $53 \%, 62 \%, 74$ and $52 \%$ respectively. In regard to specific antibiotics, resistances against $S$. aureus was $88 \%$ ampicillin, $66 \%$ gentamicin, $71 \%$ chloramphenicol, $61 \%$ ceftrazone, $69 \%$ Nalidxic acid, $80 \%$ amoxicillin, $67 \%$ tetracycline, and $82 \%$ vancomycine(Table 5).

Table 5

Antimicrobial resistance among gram positive isolates at WSUTRH, 2016-2018

\begin{tabular}{|c|c|c|c|c|c|c|c|c|c|}
\hline \multirow[t]{2}{*}{ antibiotics assayed } & \multicolumn{2}{|l|}{ S.auras } & \multicolumn{2}{|c|}{ S. saprophytes } & \multicolumn{2}{|c|}{ S. pyogenes } & \multicolumn{2}{|c|}{ S. pneumonia } & \multirow{2}{*}{$\begin{array}{l}\text { Total } \\
\mathbf{R}\end{array}$} \\
\hline & S (\%) & $\mathbf{R}(\%)$ & S (\%) & $\mathrm{R}(\%)$ & $\mathrm{S}(\%)$ & R (\%) & $\mathrm{S}(\%)$ & $\mathrm{R}(\%)$ & \\
\hline Ampicillin & $4(12)$ & $29(88)$ & NA & NA & 0 & $2(100)$ & $1(100)$ & 0 & 31 \\
\hline Gentamicin & $18(34)$ & $35(66)$ & $1(25)$ & $3(75)$ & 0 & $2(100)$ & 0 & $3(100)$ & 43 \\
\hline Cloxaciline & $20(48)$ & $22(52)$ & $2(33)$ & $4(67)$ & 0 & $1(100)$ & $1(50$ & $1(50)$ & 28 \\
\hline Chlorampencol & 16(29) & $40(71)$ & 0 & $4(100)$ & 0 & 2(100) & 0 & $2(100)$ & 48 \\
\hline Ceftrazone & 28(39) & $44(61)$ & 0 & $9(100)$ & 0 & $3(100)$ & $2(40)$ & $3(60)$ & 59 \\
\hline Nalidexin & $5(31)$ & 11(69) & $2(40)$ & $3(60)$ & $1(100)$ & 0 & $1(50)$ & $1(50)$ & 15 \\
\hline Ciprofloxacilin & $70(86)$ & 11(14) & $5(55)$ & $4(45)$ & $2(75)$ & $1(25)$ & $5(100)$ & 0 & 16 \\
\hline Clindamycine & $45(67)$ & $22(33)$ & $6(75)$ & $2(25)$ & $1(50)$ & $1(50)$ & $2(50)$ & $2(50)$ & 27 \\
\hline Cotrimoxazole & $5(45)$ & $6(55)$ & 0 & $2(100)$ & 0 & 1(100) & $1(50)$ & $1(50)$ & 10 \\
\hline Amoxicillin & $2(20)$ & $8(80)$ & $1(50)$ & $1(50)$ & NA & NA & NA & NA & 9 \\
\hline Erythromycin & $22(55)$ & 18(45) & $1(25)$ & $3(75)$ & $1(50)$ & $1(50)$ & $1(100)$ & 0 & 22 \\
\hline Tetracycline & $4(33)$ & $8(67)$ & 0 & $1(100)$ & NA & NA & 0 & 1 & 10 \\
\hline Augumentin & $14(43)$ & $18(57)$ & $2(66)$ & $1(34)$ & NA & NA & NA & NA & 19 \\
\hline Cephalexine & $11(46)$ & 13(54) & $2(66)$ & $1(34)$ & NA & NA & 1 & 1 & 15 \\
\hline Vancomicine & $3(18)$ & 14(82) & $1(100)$ & 0 & NA & NA & 0 & 1 & 15 \\
\hline Total S \&R & $267(47 \%)$ & $299(53 \%)$ & 23(38) & $38(62)$ & $5(26)$ & $14(74)$ & $15(48)$ & 16(52) & $367(54.2 \%)$ \\
\hline
\end{tabular}




\section{Antimicrobials Resistance among gram negative bacteria}

Gram negative bacteria identified were, E. coli, P. aurogenous, Proteus spp, Shigellaspp, Salmonella spp and Nisseria spp. Overall resistance for all antimicrobial resistance of E. coli was $52 \%$, P. aurogenous 75\%, proteusspp 65\%, shigellaspp 66\%, salmonella 56\% and NisseriaSpp 63\% (See Table 6).

Table 6

Antimicrobial resistance among gram negative isolates at WSUTRH, 2016-2018.

\begin{tabular}{|c|c|c|c|c|c|c|c|c|c|c|c|c|c|}
\hline \multirow{2}{*}{$\begin{array}{l}\text { Antibiotics } \\
\text { assayed for } \\
\text { sensitivity }\end{array}$} & \multicolumn{2}{|l|}{ E.coli } & \multicolumn{2}{|c|}{ P. aurogenous } & \multicolumn{2}{|c|}{ Proteus spp. } & \multicolumn{2}{|c|}{ Shigellaspp } & \multicolumn{2}{|c|}{ Salmonella } & \multicolumn{2}{|c|}{ Neisseria spp. } & \multirow[t]{2}{*}{ Total R\% } \\
\hline & S (\%) & $R^{*}(\%)$ & S (\%) & $\mathrm{R}^{*}(\%)$ & $\mathrm{s}(\%)$ & $R^{*}$ & $\mathrm{~s}$ & $R^{*}$ & S & $R^{*}$ & $\mathrm{~s}$ & $R^{*}$ & \\
\hline Ampicillin & 0 & $5(100)$ & 0 & 2 & 1 & 2 & 0 & 2 & 0 & 1 & 0 & 1 & 13 \\
\hline Gentamicin & 4 & $7(63.6)$ & 1 & 2 & 3 & 1 & 1 & 1 & 1 & 1 & 2 & 1 & 13 \\
\hline Cloxaciline & 2 & $3(60)$ & 0 & 1 & 0 & 1 & 0 & 1 & 0 & 0 & 0 & 0 & 6 \\
\hline Chlorampencol & 7 & $8(53)$ & 1 & 1 & 2 & 2 & 1 & 1 & 0 & 1 & 2 & 0 & 13 \\
\hline Ceftrazone & 5 & $9(64)$ & 2 & 6 & 1 & 2 & 3 & 1 & 0 & 0 & 0 & 2 & 20 \\
\hline Nalidexin & 7 & $1(15)$ & 1 & 3 & 1 & 1 & 0 & 1 & 1 & 0 & 0 & 0 & 6 \\
\hline Ciprofloxacilin & 12 & $4(25)$ & 2 & 6 & 2 & 2 & 2 & 2 & 0 & 0 & 1 & 2 & 16 \\
\hline Clindamycine & 3 & $7(70)$ & 1 & 6 & 0 & 2 & 0 & 2 & 0 & 1 & 1 & 0 & 18 \\
\hline Cotrimoxazole & 1 & $6(86)$ & 0 & 0 & 0 & 0 & 1 & 1 & 0 & 0 & 0 & 1 & 8 \\
\hline Amoxicillin & 0 & $3(100)$ & 1 & 0 & 0 & 1 & 0 & 0 & 0 & 0 & 0 & 0 & 4 \\
\hline Erythromycin & 5 & $0(0)$ & 1 & 4 & 0 & 1 & 0 & 0 & 1 & 0 & 0 & 1 & 6 \\
\hline Tetracycline & 1 & $2(70)$ & 0 & 1 & 0 & 2 & 0 & 2 & 0 & 1 & 0 & 1 & 9 \\
\hline Augumentin & 4 & $3(42)$ & 1 & 0 & 0 & 1 & 0 & 0 & 0 & 0 & 0 & 1 & 5 \\
\hline Cephalexine & 4 & $1(20)$ & 0 & 1 & 0 & 0 & 0 & 1 & 1 & 0 & 0 & 0 & 3 \\
\hline Vancomicine & 1 & $1(50)$ & 0 & 0 & 0 & 0 & 0 & 0 & 0 & 0 & 0 & 0 & 1 \\
\hline Total S \&R & $56(48)$ & $60(52)$ & $11(25)$ & $33(75)$ & 10(35) & $18(65)$ & $8(34)$ & $15(66)$ & $4(44)$ & $5(56)$ & $6(37)$ & $10(63)$ & $141(60 \%)$ \\
\hline
\end{tabular}

\section{Discussion}

In present study, antimicrobial resistance of gram positive isolates was $54.2 \%$ and gram negative bacteria were $60.0 \%$ where as overall antimicrobial resistance in this study was $57.1 \%$. S. auras was $92(61.3 \%)$ the most prevalent isolation from all bacteria growth and E.coli $19(12.7 \%)$ was the second isolation. Antibiotics resistance of S. auras was $53 \%$, S. saprophytes $62 \%$, S. pyogenes $74 \%$ S. pneumonia $52 \%$ and that of E. coli was $52 \%$. P. aurogenous 75\%, Proteus spp. 65\%, Nisseriaspp 63\%, salmonnela 53\%.

The finding of the antimicrobial resistance in the present study was lower than study conducted in Ethiopia, DebereMarkos Referral Hospital $84.6 \%$ for gram positive and $72.2 \%$ for gram negative bacteria and southern Ethiopia University Teaching Hospitals $74.6 \%$ among gram positive and $84.0 \%$ gram negative bacteria, [14-16]. This could be due to some updates in microbiological set ups and knowledge of prescriber in choices of drugs or creation of awareness in prescriber that Ethiopia Public Health institute Made[13].

The findings of this study is higher than studies conducted in the Africa countries indicated, Benin 34.6\%, Congo 31.9\%, Togo 14.3\%, Madagascar $16.3 \%[10]$. This might be due to the difference between geographical areas.

In the present study, Staphylococcus was predominate and E. coli the next prevalent which was similar to the study conducted in Gabon, central Africa[4]. Overall resistance of E.coli in this study was $52 \%$ and meta-analysis study conducted in the Ethiopia E.coli was $45.38 \%(33.5 \%-57.7 \%)$ and the highest resistance in Addis Ababa and the lower resistance found in the Tigray 27.5\%[17].

Resistance of S.auras for all antimicrobial testing was $53 \%$ at this study which is lower than study conducted in Greek which was $88 \%[18]$. The low finding this study compared to Greek was that this, the sample isolation was skin infection which is increased normal flora.

\section{Strength and limitation of the study}

For the nature of retrospective study, detail information of socio demographic and clinical of patients was not incorporated. The number of drug tested on some pathogens was small in number which indicated high or lower resistance. 


\section{Conclusion and recommendation}

The high prevalent bacteria pathogens isolated were $S$. auras from gram positive and of negative $E$. coli. Most of pathogens isolated showed high resistance of antimicrobial against ampicillin, gentamicin, chloramphenicol, ceftrazone, Nalidxic acid, amoxicillin, tetracycline, and vancomycine. Therefore, the study indicating collaboration among clinicians, laboratory personnel and pharmacy professionals to be guided all investigation of infectious diseases should be based on the culture and antimicrobial Sensitivity testing.

Abbreviations (Acronyms And Abbreviations)

AMR, antimicrobial resistance; ATCC, American Type Culture collection; MDR, multidrug resistance; SNNPR, South nations nationalities and peoples region; WHO, world health organizations; WSUTRH,Wolaita Sodo University Teaching Referral Hospital.

Declarations

\section{Availability of data \& materials}

The data for this research is available, so we can contact you when you need our data for the future process.

\section{Ethics approval and consent to participate}

The ethical clearance was obtained from the Ethical review committee of Wolaita Sodo University, College of Health Science and medicine. Formal permission was also obtained from Wolaita Sodo University Referral Hospital. For reasons of privacy, all data were kept confidential. Anonymity of records was maintained by using registration number and unique code numbers used by service providers at Wolaita Sodo University teaching referral hospital.

\section{Consent for publication}

Not applicable

\section{Competing interests}

The authors have declared that no competing interests exist.

\section{Funding}

This study is not funded

\section{Authors' contribution}

TA, HS, TS, TB and TL

These authors equally contributed to this research work

\section{Acknowledgment}

We would like to forward our gratitude to Wolaita Sodo University, College of Health Sciences and Medicine. We also thank Data Collectors.

\section{References}

1. WHO. WHO Report on Surveillance of Antibiotic Consumption 2016-2018 Early implementation. Report, 2018.

2. Commission E. Staff Working Paper of the Services of the Commission on

AntimicrobialResistance.Availableonline:http/ec.europa.eu/food/food/biosafety/salmonells/ antimicrobial resistance. Food and Biosafety, 2014.

3. Moges F, Mulu EM, Tessema A, Belyhun B, Shiferaw Y. Y, et al., The growing challenges of antibacterial drug resistance in Ethiopia.. 2014. doi:10.1016/j.jgar.2014.02.004. JGAR, 2014.

4. Alabi AS, et al., Retrospective analysis of antimicrobial resistanceand bacterial spectrum of infection in Gabon, Central Africa. BMC, 2013. 
5. WHO., Antimicrobial resistance (WHO Fact sheet). Geneva: World Health Organization; February 2018 (http://www.who.int/en/newsroom/factsheets/detail/antimicrobial-resistance,accessed 25 September 2018). WHO fact sheet, 2018.

6. Bank W. The World Bank. Drug resistant infections: a threat to our economic future. Washington D.C:TheWorldBank;2017(http://documents.worldbank.. Final reoprt, 2018.

7. Founou RC, Essack FL. SY, Clinical and economic impact of antibiotic resistance in developing countries: A systematic review and metaanalysis. PLoS ONE 12(12, 2017.

8. WHO. ANTIMICROBIAL RESISTANCE Global Report on Surveillance Report, 2014.

9. UNGA. AMR was the 4th health agenda that followed a Political Declaration. 2016.

10. Peggy S, Lai LMB, Meney C, Valeri L, Michelle C, White, Epidemiology of antibiotic-resistant wound infections from six countries in Africa.. BMJ Glob Health, 2018.

11. WangaiFK M. LuleGN,Karari EM,MaritimMC,JaokoWG,etal, Bridging antimicrobial resistance knowledge gaps:The East African perspective on a global problem. PLoSONE, 2019.

12. Seboxa T, Abebe AW, Tsegaye W, Hailu AAT W, et al., High Mortality from Blood Stream Infection in Addis Ababa, Ethiopia, Is Due to Antimicrobial Resistance. PLoS ONE 2015: p. 10.

13. Institute EpH, Ethiopia Antimicrobial Resistance Surveillance. surveillance, 2018.

14. Wondemagegn Mulu BA, Yimer M, Hailu T, Ayele H, Abate D. Bacterial agents and antibiotic resistance profles of infections from diferent sites that occurred among patients at Debre Markos Referral Hospital, Ethiopia: a cross-sectional study. BMC, 2017.

15. al Ae, The burden of antimicrobial resistance at tertiary care hospital, southern Ethiopia: a three years' retrospective study. BMC Infectious Diseases 2019.

16. Solomon FB, F.W.W., Amsalu Amache Arota and Yishak Leka Abraham, Antibiotic resistant airborne bacteria and their multidrug resistance pattern at University teaching referral Hospital in South Ethiopia. Ann Clin Microbiol Antimicrob (2017) 16:29 2017.

17. Kald B, Tuem AKG. Tesfay Mehari Atey ,Helen Bitew, and a.D.F.B. Ebrahim M. Yimer, Drug Resistance Patterns of Escherichia coli in Ethiopia: A Meta-Analysis. BioMed Research International, 2018: p. 13.

18. Christina Stefanak Al, Matoula T, CC,Evaggelia Polythodoraki, Chryssou S-E, George Kontochristopoulos and Christina Antoniou, Six-Year Retrospective Review of Hospital Data on Antimicrobial Resistance Profile of Staphylococcus aureus Isolated from Skin Infections from a Single Institution in Greece. antibiotics 2017.

\section{Figures}

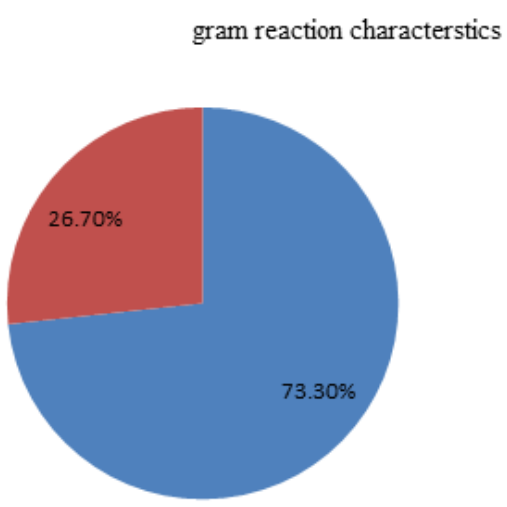

gram positive bacteria

- Gram Negative bacteria

\section{Figure 1}

Isolated pathogenic bacteria gram reaction characteristics at WSUTRH, 2016-2018 